\title{
El impacto de los estilos de liderazgo en los centros de educación media del Ecuador, su perspectiva de líderes y docentes en el año 2018
}

The impact of leadership styles in Ecuador's middle schools, their perspective of leaders and teachers in 2018

Steffy Milly Garcia Puga. ${ }^{1}$ \& Eduardo Javier Espinoza Solis. ${ }^{2}$

DOI: https://doi.org/10.33262/visionariodigital.v3i3.666

\begin{abstract}
This article refers to leadership studies and their impact on middle schools in Ecuador, which in one way or another have progressed over the years, transferring from business to other spectra of society such as education Despite this, there are still few related contributions in countries such as Ecuador, The present study approaches, from a descriptive perspective, the styles of leadership used by the leaders of secondary education centers and a comparison with the perception of their subordinate teachers. Through a quantitative methodology, 124 managers and 424 of their teachers were surveyed using the Multifactor Leadership Questionnaire (MLQ) Avolio (1995). The results show that the perceptions of leaders and subordinates are similar, with a mixed use of transformational and transactional styles predominating.
\end{abstract}

Keywords: Education, leadership, high school principals, educational centers, high school public.

\section{RESUMEN}

El presente artículo hace referencia a los estudios de liderazgo y su impacto en los centros de educación media del Ecuador, que de una u otra forma han progresado a lo largo de los

\footnotetext{
${ }^{1}$ Investigador Independiente, Milagro, Ecuador, steffymillygarcia@ gmail.com

${ }^{2}$ Universidad Estatal de Milagro, Milagro, Ecuador, eespinozas@unemi.edu.ec
} 
años, transfiriéndose del ámbito empresarial hacia otros espectros de la sociedad como la educación, pese a ello, existe aún escasos aportes relacionados en países como Ecuador. El presente estudio aborda, desde una perspectiva descriptiva, los estilos de liderazgo utilizados por los líderes de centros de educación media y un comparativo con la percepción de sus docentes subordinados. A través de una metodología cuantitativa, 124 directivos y 424 de sus docentes fueron encuestados utilizando el Cuestionario Multifactorial de Liderazgo (MLQ) Avolio (1995). Los resultados presentan que las percepciones de líderes y subordinados son similares, predominando un uso mixto de los estilos transformacional y transaccional.

Palabras Clave: educación, liderazgo, directores escolares, centros de educación, colegios públicos

\section{Introducción}

El presente artículo tiene un impacto significativo en la administración de los centros de educación media en el Ecuador una investigación basada en el impacto de los estilos de liderazgos. El liderazgo y sus primeros estudios no se originaron en el campo educativo sino en el empresarial (Flores, 2014).Ocasionando que todo lo relacionado en materia de liderazgo dentro de las instituciones de educación no fuera visto de la mejor manera por directivos y docentes, sin embargo, tal perspectiva ha cambiado considerablemente dado que el liderazgo en la actualidad es considerado como uno de los principales elementos para dinamizar, apoyar y animar el buen funcionamiento de la educación (Bolívar, 2010; Pascual, Villa, \& Auzmendi, 1993)

Así, las principales investigaciones llevadas a cabo sobre liderazgo en instituciones de educación media se han realizado en países desarrollados y anglosajones, sin embargo recientemente han empezado a surgir estudios sobre liderazgo en realidades latinoamericanas(Rivera \& Cavazos, 2007). Por tanto, aunque existen múltiples investigaciones de los estilos de liderazgo en el ámbito educativo (Castro \& Nader, 2004; Flores, 2014; Pedraja, Rodríguez, \& Rodríguez, 2016; Rojas \& Gaspar, 2006; Villa, 2015).Aún no ha sido evidenciada una investigación sobre los estilos de liderazgos presentes en la educación media en el Ecuador que exprese como se ven los directivos 
a sí mismos y que percepción tienen los docentes de ellos de forma análoga a los estudios realizados por Carchi y Vega \& Zavala (2004)en otros países.

Así, ante la ausencia de estudios previos en materia de estilos de liderazgo, se tiene como objetivo principal, identificar los estilos de liderazgo entre los directivos y la percepción de los docentes hacia sus líderes en la educación media en el Ecuador, con el presente estudió se conocerá los tipos de liderazgos existentes con base la teoría de Bass \& Avolio (1994)quienes han identificado tres principales estilos de liderazgo: el transformacional, el transaccional y el pasivo evitativo.

Se debe agregar que, para alcanzar dicho objetivo es necesario investigar y describir ciertos aspectos como los estilos de liderazgo utilizados por los directivos de los centros de educación media, identificar la percepción de los docentes sobre el estilo de liderazgo utilizado por sus directivos y por último determinar las incidencias del uso de un determinado estilo de liderazgo.

A continuación, se presenta la fundamentación teórica centrada en conocer los diferentes estilos de liderazgo existentes dentro del ámbito educativo; presentando luego el tipo de estudio y la metodología a emplear, donde por último se detalla el análisis de resultados y conclusiones de los hallazgos obtenidos a partir de los instrumentos de recolección aplicados.

\section{- Revisión de la Literatura}

- Estilos de Liderazgo

Antes de entrar en detalle, hay que tener en cuenta que el liderazgo es un constructo complejo y variado, existiendo una amplia variedad de teorías, definiéndolo como un desarrollo natural de influencia que acontece entre una persona, el líder y sus seguidores, coincidiendo en que este proceso puede ser expuesto a partir de determinadas particularidades y conductas del líder, por sensaciones y facultades de los subordinados y por el entorno en el cuál acontece dicho proceso(Jiménez \& Villanueva, 2018; Lupano \& Castro, 2006). 
Así, el liderazgo es el poderío hacia otra persona en una situación específica, para alcanzar metas por medio de la comunicación humana, es decir estrategias cuyo fin es guiar a las personas explorar sus sueños, anhelos y deseos(Chiavenato, 2001; Goleman, 2014).

Los estilos de liderazgo representan un análisis de gran relevancia para las organizaciones e instituciones, ya que reflejan la cultura organizacional y su vínculo con la sociedad en lo que respecta a una serie de comportamientos, relativamente basados en la forma de dirigir y que definen a una persona (Münch, 2010; Pazmiño, Beltrán, \& Gallardo, 2016), instaurando así un ambiente agradable para la mejora adecuada del talento humano, dado que los estilos de liderazgo pueden influir significativamente en el comportamiento de los trabajadores de la institución o empresa (Franco, Reyes, \& Cuadrado, 2017).

Así, a menudo se clasifican diferentes estilos de liderazgo acorde al comportamiento de los líderes, los cuales buscan o intentan influir en sus subordinados, los estilos de liderazgo más representativos son: transformacional, transaccional y liderazgo pasivo evitativo(Vidic, Burton, South, Pickering, \& Start, 2016).

El estilo transformacional trata de edificar relaciones entre las personas, haciendo énfasis en los valores y creando una visión compartida entre los miembros de la organización (Martin, 2015). Dicho estilo se compone de elementos tales como influencia idealizada, consideración individual, estimulación intelectual y motivación inspiradora (Solarte \& Martínez, 2017).En otras palabras, los líderes transformacionales reconocen las necesidades y habilidades individuales de los subordinados estimulando su desarrollo intelectual (Hamstra, Van Yperen, Wisse, \& Sassenberg, 2014). Este estilo en particular correlaciona positivamente con la efectividad organizacional, al promover la empatía entre compañeros dado que el líder asiste a los subordinados en su desarrollo y formación dentro de la organización (Socarrás, Sánchez, \& Ucrós, 2016). Como resultado, el estilo de liderazgo transformacional facilita la relación en el entorno de trabajo, lo cual conlleva a un nivel organizativo armónico para el manejo efectivo de los conflictos en las empresas, buscando a su vez un comportamiento proactivo por parte del equipo de trabajo (Odetunde, 2013). 
Por otro lado, el estilo transaccional como indica Martin (2015)enfatiza la relación de intercambio entre los líderes y sus subordinados, ya que especifica expectativas y responsabilidades considerando recompensas contingentes basadas en el logro de objetivos y gestión por excepción (activa), donde los líderes monitorean regularmente los errores de los subordinados (Hsu \& Chen, 2011). Como resultado, el liderazgo transaccional, enfatiza los objetivos individuales en oposición a los colectivos, tendiendo a reducir la cooperación dentro de los equipos. Además, el énfasis en las recompensas por parte de los líderes transaccionales comúnmente demuestra escasez de recursos o interdependencia negativa, instituyendo un contexto evaluativo haciendo que los subordinados demuestren su competencia superando a los demás (Hamstra et al., 2014).

El estilo transaccional de liderazgo conduce al éxito de las instituciones educativas a pesar de que no les otorga a los seguidores tanto poder como lo hace el liderazgo transformacional, sin embargo otorga a los seguidores un sentido de identidad y satisfacción laboral; eliminando las posibles barreras dentro de la institución incentivándolos a alcanzar objetivos predeterminados con un comportamiento constructivo y correctivo (Wahab, Rahmat, Yusof, \& Mohamed, 2016).

Finalmente, el estilo pasivo evitativo se lo identifica como el estilo menos sofisticado y el que menos ayuda ofrece para la consecución de objetivos dentro de una organización (Alanís \& Rico, 2009). Está considerado como estilo ineficiente, ya que resulta de un proceso de acontecimientos negativos; este cataloga a los subordinados como personas sin motivación, sin habilidad y sin conocimientos para ser grandes triunfadores (Vidic et al., 2016).Como consecuencia, se evidencia una ausencia total de liderazgo, es decir un estilo en el que un líder esquivado evade sus responsabilidades y solo actúa cuando es forzado. Es posible que este tipo de "líder" ya no se interese o no tenga confianza en su capacidad para dirigir (Martin, 2015).

A medida de que las organizaciones se vuelven más grandes y complejas, es imprescindible estudiar el estilo de liderazgo, los conocimientos, habilidades y/o experiencia requeridos al momento de implementarlo en escenarios oportunos y el nivel de motivación intrínseca que necesita el líder para utilizar cada estilo, así como su capacidad para infundirla en sus 
subordinados con base a los múltiples factores que posee cada estilo de liderazgo(Vidic et al., 2016).

En resumen, la investigación y sus resultados se pueden llegar a considerar como directrices a seguir por parte de directivos y docentes de las instituciones educativas, buscando conocer que estilo de liderazgo predomina y permitiendo así ampliar los conceptos acerca del liderazgo directivo en la educación, adentrándose por lo tanto en la búsqueda de tendencias que influyan en el buen rendimiento de los funcionarios, teniendo claro que cada estilo de liderazgo tendrá diferentes resultados dentro de la educación media, siendo así los estudiantes quienes finalmente podrían ser o no los beneficiados (Villalobos, Peña, Aguirre, \& Guerrero, 2017).

\section{Percepción de los colaboradores}

Así, en lo que respecta a los estilos de liderazgo, la investigación iniciada en 1945 por The Ohio State University, demostró que los subordinados veían el comportamiento del estilo de liderazgo, en términos de dos dimensiones: la primera manifiesta el grado de orientación de la tarea del líder, es decir, la medida en que ella delimita y estructura el trabajo de los subordinados, mientras tanto la segunda se refiere al grado de orientación de la relación del comportamiento de un líder, que es la medida en que muestra preocupación por los subordinados y vela por su bienestar (Jost, 2013).

\section{Estilos de liderazgo en el ámbito educativo}

$\mathrm{Al}$ momento, se han intensificado las expectativas y los requerimientos en lo que respecta al rol de directivos, administradores, entre otros., del manejo de instituciones de educación, observando la necesidad de saber capacidades y características del "liderazgo educativo", abriendo el campo de discusión de conceptos claves de dicho rol, particularmente visto desde una óptica de relaciones entre directores (en un esquema de autoevaluación) y docentes (esquema de evaluación a terceros o líderes) (Volante, Díaz, \& Tapia, 2003).Así, el liderazgo, en el ámbito educacional, establece el valor de interacción entre dos o más miembros de un grupo, donde habitualmente se estructura o reestructuran percepciones y expectativas de sus conductas (Ganga \& Navarrete, 2013; Willman \& Velasco, 2011), es decir el patrón de comportamiento que los 
líderes utilizan en el trabajo, destacando habilidades y actitudes que revelan el carácter y conducta de una persona; haciendo además hincapié a la disposición de los líderes a tomar en cuenta sugerencias de los subordinados para la toma de decisiones, descentralizando la autoridad y reconociendo de esta manera la capacidad de los demás en el desarrollo de ideas y actividades (Solarte \& Martínez, 2017; Yamak \& Eyüpoğlu, 2018).

De ahí que, el liderazgo se ha convertido en la actualidad en una prioridad dentro del ámbito educativo, sobre todo en los programas de política educativa a nivel internacional (Bolívar, 2010). Por lo tanto, en un mundo globalizado y cambiante, los líderes educativos tienen el desafío de encontrar los estilos de liderazgo que mejoren las técnicas de instrucción hacia los docentes y a su vez continuar con su desarrollo profesional, enfocando así su atención y la de toda la comunidad educativa en el aprendizaje (Mehdinezhad \& Sardarzahi, 2016). Además, la educación media probablemente es una condición necesaria, pero no suficiente para nutrir el liderazgo del desarrollo y habilidades (Schweisfurth, Davies, Symaco, \& Valiente, 2018).

De tal manera, las características del personal directivo en la educación y sus estilos de liderazgo, son las variables que determinarán el proceso de toma de decisiones y sus resultados, donde, al contar con un equipo directivo no sólo que influenciará en conductas de los docentes sino que serán concluyentes para la dirección de actividades, consecución de metas e instauración de un orden y disciplina, ayudando así al desarrollo a largo plazo de las instituciones educativas(Mehdinezhad \& Sardarzahi, 2016; Rodríguez, 2006), para lo cual, uno de los estilos de liderazgo que se adapta a este escenario es el transformacional, el mismo que indica que los líderes delegan y comparten su autoridad para tomar decisiones con su equipo de trabajo (Yamak \& Eyüpoğlu, 2018).Por ejemplo, el liderazgo transformacional permite a los directivos involucrar al personal haciéndolos sentir como un activo de valor para el establecimiento educativo; además este estilo de liderazgo aumenta la capacidad del director para estimular a los empleados intelectualmente para desafiar el 'status quo' y desarrollar soluciones creativas e innovadoras a los problemas (Aina, 2016). Además los líderes transformacionales motivan a sus seguidores a ser plenamente conscientes de la importancia de los resultados de sus tareas y los inducen a superar sus propios intereses por el bien de las organizaciones al lograr sus mayores necesidades (Elrehail, Emeagwali, Alsaad, \& Alzghoul, 2018). 
Así, Leal, Albornoz, \& Rojas (2016) en su estudio evidenciaron que el liderazgo transformacional tiene mayor presencia, seguido del liderazgo transaccional por último el liderazgo pasivo evitativo la presencia más baja de acuerdo a sus resultados. Además, el liderazgo transformacional correlaciona significativamente fuerte y directamente proporcional con el liderazgo transaccional por lo que interpretan que, a una mayor manifestación de liderazgo transformacional, se genera un mayor liderazgo transaccional, sin embargo, el liderazgo pasivo evitativo correlaciona negativamente e inversamente proporcional con los liderazgos transaccional y transformacional, es decir a manera que aumenta el liderazgo transformacional y transaccional, el liderazgo pasivo evitativo disminuye(Pedraja, Rodríguez, Barreda, Sagredo, \& Segovia, 2009).

Como resultado, en cuanto a la relación con los estilos de liderazgo dentro de los entornos de educación media, muchos líderes académicos prefieren el liderazgo transformacional por cuanto tiene más probabilidades de sostener el cambio del sistema educativo (Wahab et al., 2016). Dicho liderazgo se enfoca en reestructurar la institución educativa media mejorando las condiciones escolares, de los practicantes destacados y de los estudiantes de liderazgo (Cetin \& Kinik, 2015).

Aunque, es necesario recalcar que no siempre en el ámbito educativo el liderazgo transaccional es menor que el transformacional, ya que existen ocasiones que ambos liderazgos están presentes en el ámbito educativo por igual, así lo demuestran Romero y Sidro (2016) en su investigación a directores de escuelas, donde los promedios de los liderazgos transaccionales y transformacionales son semejantes, en otras palabras los directores ejercen ambos liderazgos combinados sin que predomine alguno en particular.

En ocasiones el liderazgo transaccional puede tener mayor presencia que el liderazgo pasivo evitativo y transformacional, así lo demuestran Javed, Jaffari, \& Rahim (2014) en un estudio realizado en el ámbito educativo donde se evidenció que el liderazgo transaccional era percibido de mayor manera, en comparación con el liderazgo transformacional; como resultado el estilo transaccional ejecuta un sistema de reclutamiento y la preparación individualizada de los empleados, con énfasis en la superación personal y la obtención de resultados de manera 
independiente (Mehdinezhad \& Sardarzahi, 2016), a la vez, tienen un desempeño en habilidades tales como la explotación de recursos, la innovación y la responsabilidad (Afshari et al., 2017).

Así, los estilos de liderazgo de los directores experimentados e inexpertos son relativamente iguales y generalmente aceptables (Mehdinezhad \& Sardarzahi, 2016), pero esto en muchas ocasiones tiende a demostrar un liderazgo ineficaz produciendo estrés en el personal, un rendimiento deficiente y una alta rotación del mismo, lo que traduce en problemas para las instituciones educativas (Yamak \& Eyüpoğlu, 2018). Por ejemplo, el liderazgo pasivo evitativo se puede presentar en algunas instituciones y donde en ocasiones su promedio es similar con los liderazgos transaccional o transformacional, así lo indican en su estudio Molero, Recio, \& Cuadrado (2010) donde aun cuando los liderazgos transformacional y transaccional tiene una mayor presencia, el liderazgo pasivo evitativo muy semejante. Además, las correlaciones del liderazgo pasivo evitativo también van casi a la par salvo que, en sentido inversamente proporcional con los liderazgos transformacional y transaccional.

En pocas palabras, las instituciones de educación media deben basarse en una gestión escolar y liderazgo eficaz a partir de la evolución de los recursos humanos, con un enfoque no solamente transformador, sino integrador de todos los elementos que intervienen en el proceso educativo (Rivera \& Cavazos, 2007). Donde el estilo de liderazgo que predomine en las instituciones será referente en los procesos de administración y relación en los procesos de aprendizaje, lo cual incrementará su presencia, calidad y liderazgo (Escobedo \& Manuel, 2014), poniendo a los directivos abiertos a nuevas ideas (Sperber \& Linder, 2018).

Hipótesis 1. Los líderes de las instituciones educativas tienen una percepción de sí mismos de utilizar mayormente el estilo de liderazgo transformacional que transaccional y pasivo evitativo. Hipótesis. 2 los líderes que se perciben como transformacionales o transaccionales reducen su uso del estilo pasivo evitativo.

Hipótesis. 3 los docentes de los centros educativos perciben a sus líderes mayormente como transformacionales que transaccionales y pasivo evitativo. 


\section{METODOLOGÍA}

De acuerdo a los objetivos trazados, la presente investigación corresponde a un estudio de carácter descriptivo y documental, además la investigación posee un enfoque cuantitativo al ser necesario recolectar información que permita a través de mediciones de las diferentes variables comprobar las hipótesis(Hernández, Fernández, \& Baptista, 2014; Villacorta, 2015).

De este modo, la técnica de recolección de datos utilizada dentro de la investigación fue la encuesta, la cual a consideración de Hernández et al. (2014), está destinada a obtener datos de varias personas, en este caso de los funcionarios de los centros de educación antes mencionados, cuyas opiniones son de interés, de manera que permita conocer e interpretar criterios y apreciaciones sobre el tema.

\section{Muestra}

La muestra estuvo compuesta por $(\mathrm{N}=124)$ directivos y $(\mathrm{N}=424)$ docentes subordinados de centros de educación media de la región 5 del Ecuador, considerando que sean mayores de edad, se encuentren en funciones al momento de contestar el instrumento. La recogida de información se realizó en el periodo comprendido entre los meses de junio y julio del 2018.

\section{Instrumento}

Para la recolección de datos se tomó el cuestionario planteado por Avolio \& Bass (1995)quienes han trabajado sobre el paradigma transformacional - transaccional con el desarrollo del cuestionario, MLQ - Cuestionario Multifactorial de Liderazgo. El cuestionario contiene 45 preguntas, permitiendo medir hasta qué punto un líder es transformacional, transaccional o pasivo evitativo. El cuestionario proporciona, a través de una escala de Likertcon respuestas que van de 1 a 5, información sobre diferentes estilos de liderazgo. Un ejemplo para liderazgo transformacional es "hablo del futuro con optimismo", para liderazgo transaccional "dedico tiempo a la enseñanza y a la formación "y para liderazgo pasivo evitativo "evito tomar decisiones", al instrumento se agregaron también datos demográficos que permitirán obtener mayores variables de análisis. 
ISSN: 2602-8506

Por último, para el tratamiento de los datos se utilizó el software estadístico SPSS 23.0, el cual posibilitó la cuantificación de las variables y determinar la relación de las mismas a través de múltiples análisis.

\section{Resultados}

Una vez analizados los datos se ha podido evidenciar que en la educación media del Ecuador existe una notable diferencia de género en lo que respecta a sus líderes como se observa en la tabla 1, existiendo un $20 \%$ de hombres y un $80 \%$ de mujeres. Además, se puede evidenciar que la edad comprendida entre 35 y 55 años es la que mayor predomina entre los directivos con alrededor del $64 \%$ y donde el nivel de estudio de mayor porcentaje es el de tercer nivel, el cual lo posee alrededor del $81 \%$, seguido de los estudios de cuarto nivel con el $18 \%$, por último, la mayoría de los directivos de la educación media del Ecuador, el 75\%, tiene de 1 a 5 años de antigüedad en puestos de dirección.

Tabla 1. Demográficos Directivos

\begin{tabular}{|c|c|c|c|c|}
\hline & & Recuento & $\begin{array}{l}\% \text { de } \mathrm{N} \\
\text { tablas }\end{array}$ & $\begin{array}{r}\text { Desviación } \\
\text { estándar }\end{array}$ \\
\hline \multirow[t]{3}{*}{ Género } & Masculino & 27 & $21,8 \%$ & \\
\hline & Femenino & 97 & $78,2 \%$ & \\
\hline & Total & 124 & $100,0 \%$ & ,41 \\
\hline \multirow[t]{4}{*}{ Edad } & Menor a 35 años & 30 & $24,2 \%$ & \\
\hline & Entre 35 y 55 años & 79 & $63,7 \%$ & \\
\hline & Mayor a 55 años & 15 & $12,1 \%$ & \\
\hline & Total & 124 & $100,0 \%$ &, 59 \\
\hline \multirow{4}{*}{$\begin{array}{c}\text { Tipo de institución que } \\
\text { dirige }\end{array}$} & Pública & 123 & $99,2 \%$ & \\
\hline & Privada & 1 & $0,8 \%$ & \\
\hline & Total & 124 & $100,0 \%$ & ,09 \\
\hline & Segundo Nivel & 2 & $1,6 \%$ & \\
\hline \multirow{3}{*}{ Nivel de estudios } & Tercer Nivel & 100 & $80,6 \%$ & \\
\hline & Cuarto Nivel & 22 & $17,7 \%$ & \\
\hline & Total & 124 & $100,0 \%$ & ,41 \\
\hline Antigüedad en la & 1 a 5 años & 53 & $42,7 \%$ & \\
\hline
\end{tabular}


ISSN: 2602-8506

Vol. 3, N³., p.134-156, julio - septiembre, 2019

\begin{tabular}{clccc}
\hline institución & 5 a 10 años & 34 & $27,4 \%$ & \\
& Más de 10 años & 37 & $29,8 \%$ & \\
& Total & 124 & $100,0 \%$ &, 85 \\
Antigüedad en puestos & 1 a 5 años & 93 & $75,0 \%$ & \\
de dirección & 5 a 10 años & 13 & $10,5 \%$ & \\
& Más de 10 años & 18 & $14,5 \%$ & \\
& Total & 124 & $100,0 \%$ &, 73 \\
De cuantas personas es & Menor a 5 & 64 & $51,6 \%$ & \\
usted jefe o Supervisor & Entre 6 y 20 & 28 & $22,6 \%$ & \\
& Mayor a 20 & 32 & $25,8 \%$ &, 84 \\
\hline
\end{tabular}

En otro aspecto, La tabla 2 evidencia que existe una mayor autopercepción del liderazgo transformacional con una media de 4.51

Tabla 2. Estilos de liderazgos. Autopercepción de Directivos en la educación media del Ecuador

\begin{tabular}{lcccccc}
\hline & $\begin{array}{c}\text { Masculino } \\
\text { Media }\end{array}$ & $\begin{array}{c}\text { Femenino } \\
\text { Media }\end{array}$ & Media & $\begin{array}{c}\text { Total } \\
\text { Desviación } \\
\text { estándar }\end{array}$ & Varianza \\
\hline Influencia Idealizada atribuida & 4,26 & 4,46 & 4,42 &, 60 &, 36 \\
Influencia idealizada conductual & 4,44 & 4,40 & 4,41 &, 53 &, 29 \\
Motivación inspiracional & 4,70 & 4,81 & 4,79 &, 36 &, 13 \\
Carisma Inspiracional & 4,47 & 4,56 & 4,54 &, 37 &, 14 \\
Estimulación intelectual & 4,37 & 4,42 & 4,41 &, 53 &, 28 \\
TRANSFORMACIONAL & 4,44 & 4,52 & 4,51 &, 37 &, 14 \\
Consideración individualizada & 4,25 & 4,35 & 4,33 &, 54 &, 29 \\
Recompensa contingente & 4,35 & 4,30 & 4,31 &, 66 &, 43 \\
TRANSACCIONAL & 4,30 & 4,33 & 4,32 &, 45 &, 20 \\
Dirección excepción activa & 3,78 & 4,06 & 4,00 &, 73 &, 54 \\
Dirección excepción pasiva & 1,49 & 1,46 & 1,47 &, 61 &, 37 \\
Laissez faire & 2,33 & 2,29 & 2,30 &, 55 &, 30 \\
PASIVO EVITATIVO & 1,91 & 1,88 & 1,89 &, 51 &, 26 \\
\hline
\end{tabular}


ISSN: 2602-8506

Vol. 3, N³., p.134-156, julio - septiembre, 2019

No obstante, aunque exista una mayor presencia del liderazgo transformacional, se evidencia que

el liderazgo transaccional está casi a la par con un valor promedio de 4.32Igualmente, se observa que el liderazgo pasivo evitativo tiene menor presencia, con una media de 1.91. Con esto podemos corroborar la hipótesis 1 .

Por otra parte, en la tabla 3 se establecieron correlaciones entre los estilos de liderazgo, en cuanto al liderazgo transformacional se observa que correlaciona significativamente fuerte $\mathrm{y}$ directamente proporcional con el liderazgo transaccional con 0.604 .

Tabla 3. Correlaciones. Autopercepción de Directivos en la educación media del Ecuador

\begin{tabular}{lcccc}
\hline & & Transformacional & Transaccional & $\begin{array}{c}\text { Pasivo } \\
\text { evitativo }\end{array}$ \\
\hline Transformacional & Correlación de & 1 &, $604^{* *}$ &,- 095 \\
& Pearson & & &, 000 \\
Sig. (bilateral) & N & 124 & 124 & 124 \\
Transaccional & Correlación de &, $604^{* *}$ & 1 &, 002 \\
& Pearson & & & \\
& Sig. (bilateral) &, 000 & 124 &, 979 \\
& N & 124 &, 987 &, 000 \\
Sig. (bilateral) &, 176 & 124 & 124 \\
evitativo & N & 124 &, 002 & 1 \\
& Correlación de &,- 095 & & \\
& Pearson & &, 979 & 124 \\
\hline & Sig. (bilateral) &, 293 & 124 & \\
\hline & N & 124 & & \\
\hline
\end{tabular}

Además, se evidencia que el liderazgo pasivo evitativo correlaciona, pero no de manera significativa e inversamente proporcional con el liderazgo transformacional con -0.095 , a la vez se demuestra que dentro de la autopercepción de los directivos destaca una correlación positiva pero insignificante entre el liderazgo pasivo evitativo y el liderazgo transaccional con 0.002 . 
ISSN: 2602-8506

Se observa que pocos líderes manejan un estilo de liderazgo pasivo evitativo. Se evidencia entonces que existen patrones de comportamiento en la autopercepción de los líderes que no dicen que sean exclusivamente transformacionales o transaccionales, encontrándose que hay algunos de ellos que demuestran que son líderes mixtos, por lo que la hipótesis 2 es corroborada.

\section{Percepciones de los docentes hacia sus líderes}

Los demográficos de esta muestra presentan que existe una notable diferencia de género en ella como se observa en la tabla 4 , existiendo un $25 \%$ de hombres y un $75 \%$ de mujeres, la edad comprendida entre 35 y 55 años es la que mayor predomina con el 65\%, y donde el nivel de estudio de mayor porcentaje es el de tercer nivel, el cual lo posee alrededor del $80 \%$.Por último, la mayoría de los docentes el 75\% tiene de 1 a 5 años de antiguiedad en la institución.

Tabla 4. Demográficos Docentes

\begin{tabular}{|c|c|c|c|c|}
\hline & & Recuento & $\begin{array}{l}\% \text { de } N \\
\text { tablas }\end{array}$ & $\begin{array}{r}\text { Desviación } \\
\text { estándar }\end{array}$ \\
\hline \multirow{3}{*}{ Género } & Masculino & 108 & $25,5 \%$ & \\
\hline & Femenino & 316 & $74,5 \%$ & \\
\hline & Total & 424 & $100,0 \%$ & ,44 \\
\hline \multirow{4}{*}{ Edad } & Menor a 35 años & 112 & $26,4 \%$ & \\
\hline & Entre 35 y 55 años & 278 & $65,6 \%$ & \\
\hline & Mayor a 55 años & 34 & $8,0 \%$ & \\
\hline & Total & 424 & $100,0 \%$ &, 56 \\
\hline \multirow{4}{*}{$\begin{array}{l}\text { Tipo de } \\
\text { institución a la } \\
\text { que pertenece }\end{array}$} & Pública & 422 & $99,5 \%$ & \\
\hline & Privada & 2 & $0,5 \%$ & \\
\hline & Total & 424 & $100,0 \%$ &, 07 \\
\hline & Segundo Nivel & 17 & $4,0 \%$ & \\
\hline \multirow{3}{*}{ Nivel de estudios } & Tercer Nivel & 340 & $80,2 \%$ & \\
\hline & Cuarto Nivel & 67 & $15,8 \%$ & \\
\hline & Total & 424 & $100,0 \%$ & ,43 \\
\hline \multirow{4}{*}{$\begin{array}{l}\text { Antigüedad en la } \\
\text { institución }\end{array}$} & 1 a 5 años & 233 & $55,0 \%$ & \\
\hline & 5 a 10 años & 79 & $18,6 \%$ & \\
\hline & Más de 10 años & 112 & $26,4 \%$ & \\
\hline & Total & 424 & $100,0 \%$ &, 86 \\
\hline
\end{tabular}


En otro aspecto, los ven a sus líderes como más transformacionales según se aprecia en la tabla 5, con una media de 3.91 No obstante, sucede exactamente lo mismo que con la autopercepción de sus líderes, reflejando un estilo liderazgo transaccional casi a la par del transformacional, con una media de 3.74, Además, visto desde la percepción de los docentes el liderazgo pasivo evitativo tiene muy poca presencia con una media de 2.63. De este modo la hipótesis 3 es corroborada.

Tabla 5. Estilos de liderazgos. Percepción de Docentes hacia sus líderes en la educación media del Ecuador

\begin{tabular}{|c|c|c|c|c|c|}
\hline & Masculino & Femenino & Media & $\begin{array}{r}\text { Total } \\
\text { Desviación } \\
\text { estándar }\end{array}$ & Varianza \\
\hline Influencia Idealizada atribuida & 3,90 & 3,71 & 3,76 & 1,06 & 1,12 \\
\hline Influencia idealizada conductual & 4,08 & 3,86 & 3,92 & 1,02 & 1,05 \\
\hline Motivación inspiracional & 4,31 & 4,11 & 4,16 & 1,08 & 1,16 \\
\hline Carisma Inspiracional & 4,09 & 3,89 & 3,94 & 1,00 & ,99 \\
\hline Estimulación intelectual & 4,01 & 3,76 & 3,82 & 1,03 & 1,07 \\
\hline TRANSFORMACIONAL & 4,07 & 3,86 & 3,91 & ,98 & ,96 \\
\hline Consideración individualizada & 3,75 & 3,63 & 3,66 & ,86 &, 75 \\
\hline Recompensa contingente & 4,01 & 3,75 & 3,81 & ,99 & ,98 \\
\hline TRANSACCIONAL & 3,88 & 3,69 & 3,74 & ,86 & ,74 \\
\hline Dirección excepción activa & 3,87 & 3,63 & 3,69 & ,98 & ,97 \\
\hline Dirección excepción pasiva & 1,77 & 1,81 & 1,80 & ,86 & ,74 \\
\hline Liderazgo pasivo evitativo & 2,26 & 2,34 & 2,32 & 60 & ,36 \\
\hline Liderazgo correctivo evitativo & 2,01 & 2,07 & 2,06 & ,66 & ,44 \\
\hline PASIVO EVITATIVO & 2,63 & 2,59 & 2,60 & ,42 &, 18 \\
\hline
\end{tabular}

De lo anterior mencionado, en el presente estudio se destaca que los docentes ven a sus líderes con una mayor presencia del liderazgo pasivo evitativo en comparación con la autopercepción de los líderes, es decir una vez completado ambos cuestionarios tanto el de autopercepción (líderes) como el de percepción (docentes) arrojan promedios de 1.89 y 2.63 respectivamente.

En otro aspecto, la percepción de los docentes hacia sus líderes, denota que el liderazgo transformacional correlaciona significativamente fuerte y proporcional con el liderazgo 
ISSN: 2602-8506

Vol. 3, N³., p.134-156, julio - septiembre, 2019

transaccional como se observa en la tabla 6, correspondiendo a un valor de correlación de 0.904 , Igualmente, los resultados demuestran que el liderazgo pasivo evitativo correlaciona, de manera significativa e inversamente proporcional con el liderazgo transformacional con -0.552 al igual que con el liderazgo transaccional con -0.442

Tabla 6. Correlaciones. Percepción de Docentes hacia sus líderes en la educación media del Ecuador

\begin{tabular}{|c|c|c|c|c|}
\hline & & Transformacional & Transaccional & $\begin{array}{c}\text { Pasivo } \\
\text { evitativo }\end{array}$ \\
\hline \multirow{4}{*}{ Transformacional } & Correlación de & 1 & ,904** &,$- 552^{* *}$ \\
\hline & Pearson & & & \\
\hline & Sig. (bilateral) & & ,000 & ,000 \\
\hline & $\mathrm{N}$ & 424 & 424 & 424 \\
\hline \multirow{4}{*}{ Transaccional } & Correlación de &, $904^{* *}$ & 1 &,$- 442^{* *}$ \\
\hline & Pearson & & & \\
\hline & Sig. (bilateral) & ,000 & & ,000 \\
\hline & $\mathrm{N}$ & 424 & 424 & 424 \\
\hline \multirow{4}{*}{$\begin{array}{l}\text { Liderazgo pasivo } \\
\text { evitativo }\end{array}$} & Correlación de &,$- 552^{* *}$ &,$- 442^{* *}$ & 1 \\
\hline & Pearson & & & \\
\hline & Sig. (bilateral) & ,000 & ,000 & \\
\hline & $\mathrm{N}$ & 424 & 424 & 424 \\
\hline
\end{tabular}

Finalmente, los docentes y su percepción hacia sus líderes muestran consideraciones similares a la autopercepción de sus líderes, donde la única diferencia significativa es la ausencia de una correlación positiva y proporcional entre el liderazgo pasivo evitativo y el liderazgo transaccional.

\section{Discusión}

Los resultados de la presente investigación guardan relación con lo que sostienen Leal, Albornoz, \& Rojas (2016) y Pedraja et al. (2009)quienes en su estudio señalan que el liderazgo transformacional en el sector educativo tiene mayor presencia, seguido del liderazgo 
transaccional, es decir a manera que aumenta el liderazgo transformacional y transaccional, el liderazgo pasivo evitativo disminuye, sin embargo difiere del estudio Javed, Jaffari, \& Rahim (2014) quienes demuestran que el liderazgo transaccional puede tener mayor presencia que el liderazgo pasivo evitativo y transformacional.

\section{Conclusiones}

A manera de conclusión, este estudio analizó los estilos de liderazgo utilizados por los líderes de centros de educación media y la percepción de sus docentes subordinados, aportando información sobre estudios de liderazgo. Se destaca que estos directivos no utilizan un estilo de liderazgo de manera mayoritaria sino un mix entre los estilos de liderazgo transformacional y transaccional, dato fundamental que evidencia que poseen la capacidad de acoplarse dependiendo del contexto, de sus subordinados y de sus objetivos. Además, la percepción de los docentes subordinados corrobora lo enunciado por sus líderes, aunque con puntuaciones menores.

\section{BIBLIOGRAFÍA}

Afshari, J., Moein, Z., Afshari, F., Sharifi-Rad, J., Balouchi, A., \& Afshari, A. (2017). A comparison of leadership styles with respect to biographical characteristics. SA Journal of Human Resource Management, 15(0), 1-7. https://doi.org/10.4102/sajhrm.v15i0.817

Aina, O. A. (2016). Effect of leadership style on organizational commitment.

Alanís, D. C., \& Rico, M. C. (2009). Relación entre los estilos de liderazgo de los directivos del CIIDIR Unidad Durango y la percepción de su eficacia desde el enfoque del MLRC. Retos de Las Ciencias Administrativas Desde Las Economías Emergentes: Evolución de Sociedades, (2009), 1-25.

Avolio, B., \& Bass, B. M. (1995). Individual consideration viewed at multiple levels of analysis: A multi-framework for examining the diffusion of transformational leadership. Leadership Quarterly, 188-218.

Bass, B., \& Avolio, B. (1994). Improving organizational effectiveness through transformational leadership. New Delhi: SAGE Publications, Inc. 
ISSN: 2602-8506

Vol. 3, N³., p.134-156, julio - septiembre, 2019

Bolívar, A. (2010). Educational leadership and its role in improvement: A current review of its possibilities and limitations. Psicoperspectivas, 9(2), 9-33. https://doi.org/:10.5027/PSICOPERSPECTIVAS-VOL9-ISSUE2-FULLTEXT-112

Carchi, J. (2014). Estudio comparativo de los estilos de liderazgo y la percepción de los seguidores. Caso Empresa Industrial INDUGLOB. Universidad del Azuay.

Castro, A., \& Nader, M. (2004). Estilos de liderazgo, contexto y cultura organizacional. Un estudio comparativo en población Civil y Militar. Boletín de Psicología, (82), 45-63.

Cetin, M. O., \& Kinik, F. S. F. (2015). An Analysis of Academic Leadership Behavior from the Perspective of Transformational Leadership. Procedia - Social and Behavioral Sciences, 207, 519-527. https://doi.org/10.1016/j.sbspro.2015.10.122

Chiavenato, I. (2001). Administración proceso administrativo. Bogotá: McGraw-Hill Interamericana S.A.

Elrehail, H., Emeagwali, O. L., Alsaad, A., \& Alzghoul, A. (2018). The impact of Transformational and Authentic leadership on innovation in higher education: The contingent role of knowledge sharing. Telematics and Informatics, 35(1), 55-67. https://doi.org/10.1016/j.tele.2017.09.018

Escobedo, C., \& Manuel, J. (2014). Liderazgo educativo, un reto constante en educación superior. International Journal of Good Conscience Diciembre, 9(3), 119-126.

Flores, E. (2014). El Estilo de liderazgo de los directores de los colegios parroquiales de Piura. Universidad de Piura.

Franco, A. M., Reyes, M., \& Cuadrado, S. (2017). Incidencia de los Estilos de Liderazgo en la Satisfacción de los Colaboradores en Empresas de Servicios del Ecuador Incidence of Leadership Styles in Employee Satisfaction in Business Services in Ecuador. PODIUM. Edición Especial, (1390-5473), 41-64.

Ganga, F., \& Navarrete, E. (2013). Enfoques asociados al liderazgo eficaz para la organización. 
Revista Gaceta Laboral, 19(1), 52-77.

Goleman, D. (2014). Cómo ser un líder. B de Books.

Hamstra, M. R. W., Van Yperen, N. W., Wisse, B., \& Sassenberg, K. (2014). Transformational and Transactional Leadership and Followers' Achievement Goals. Journal of Business and Psychology, 29(3), 413-425. https://doi.org/10.1007/s10869-013-9322-9

Hernández, R., Fernández, C., \& Baptista, M. del P. (2014). Metodología de la investigación. México: McGraw-Hill/INTERAMERICANA EDITORES, S.A DE C.V.

Hsu, C. Y., \& Chen, W. Y. (2011). Subordinates' perception of managers' transformational leadership style and satisfaction: A comparison of electronic manufacturing companies in mainland China and Taiwan. International Journal of Human Resource Management, 22(15), 3097-3108. https://doi.org/10.1080/09585192.2011.560872

Javed, H. A., Jaffari, A. A., \& Rahim, M. (2014). Leadership Styles and Employees' Job Satisfaction: A Case from the Private Banking Sector of Pakistan. Journal of Asian Business Strategy, 41-50.

Jiménez, A., \& Villanueva, M. (2018). Los estilos de liderazgo y su influencia en la organización: Estudio de casos en el Campo de Gibraltar. Revista de La Agrupación Joven Iberoamericana de Contabilidad Y Administración de Empresas (AJOICA), 183-195.

Jost, P.-J. (2013). An economic theory of leadership styles. Review of Managerial Science, 7(4), 365-391. https://doi.org/10.1007/s11846-012-0081-1

Leal, F., Albornoz, M., \& Rojas, M. I. (2016). Liderazgo directivo y condiciones para la innovación en escuelas chilenas. Estudios Pedagógicos, 193-205.

Lupano, M. L., \& Castro, A. (2006). Estudios sobre el liderazgo. Teorías y evaluación. Psicodebate. Psicología, Cultura Y Sociedad, 19(1215), 107-122.

Martin, J. (2015). Transformational and Transactional Leadership: An Exploration of Gender, Experience, and Institution Type. Portal: Libraries and the Academy, 15(2), 331-351. 
ISSN: 2602-8506

https://doi.org/10.1353/pla.2015.0015

Mehdinezhad, V., \& Sardarzahi, Z. (2016). Leadership Behaviors and Its Relation with Principals' Management Experience. Journal of New Approaches in Educational Research, 6(1), 11-16. https://doi.org/10.7821/naer.2016.1.133

Molero, F., Recio, P., \& Cuadrado, I. (2010). Liderazgo transformacional y liderazgo transaccional : un análisis de la estructura factorial del Multifactor Leadership Questionnaire ( MLQ ) en una muestra española. Psicothema.

Münch, L. (2010). Administración Gestión organizacional, enfoques y proceso administrativo. México: Pearson Educación.

Northhouse, P. (2016). Leadership -- Theory and Practice. SAGE Publications, Inc. Michigan. https://doi.org/2016

Odetunde, O. J. (2013). Influence of Transformational and Transactional Leaderships, and Leaders' Sex on Organisational Conflict Management Behaviour. Gender \& Behaviour, 11(1), 5323-5335. https://doi.org/10.4314/gab.v11i1.

Pascual, R., Villa, A., \& Auzmendi, E. (1993). El liderazgo transformacional en los centros docentes. Bilbao: Mensajero.

Pazmiño, G., Betran, M., \& Gallardo, W. (2016). Estilos de liderazgo y su influencia en el desarrollo empresarial : Caso Pymes de la Provincia de Tungurahua -. PUCE, (1390-7719), 355-369.

Pedraja, L., Rodríguez, E., Barreda, M., Sagredo, O., \& Segovia, C. (2009). Estilos de liderazgo y resultados del sistema de medición de la calidad de la educación: Un estudio empírico en los colegios básicos de la ciudad de Arica - Chile. Revista Chilena de Ingeniería, 17, 21-26.

Pedraja, L., Rodríguez, E., \& Rodríguez, P. (2016). Estilos de liderazgo de dirección escolar Y El Logro Académico De Los Estudiantes: Un Estudio Exploratorio. INTERCIENCIA, 41(November), 748-757. 
Rivera, J., \& Cavazos, J. (2007). La importancia de la gestión y el liderazgo escolar en las instituciones de Educación Media Superior Tecnológica (CETIS y CBTIS) del estado de Tlaxcala. Revista Iberoamericana Para La Investigación Y El Desarrollo Educativo, 6.

Rodríguez, E. (2006, July). El proceso de toma de decisiones estratégicas en las universidades públicas. Revista Calidad En La Educación, 49-63. https://doi.org/https://doi.org/10.31619/caledu.n24.267

Rojas, A., \& Gaspar, F. (2006). Bases del liderazgo en educación. Oficina Regional de Educación de La UNESCO Para América Latina Y El Caribe, (1), 149. https://doi.org/10.1007/s13398014-0173-7.2

Romero, F. (2016). Estilos de liderazgo en directores de escuelas primarias públicas de la Zona 078 de Yucatán. Universidad Pedagógica Nacional.

Schweisfurth, M., Davies, L., Symaco, L. P., \& Valiente, O. (2018). Higher education, bridging capital, and developmental leadership in the Philippines: Learning to be a crossover reformer. International Journal of Educational Development, 59(October 2017), 1-8. https://doi.org/10.1016/j.ijedudev.2017.09.001

Sidro, J. (2016). Efecto del liderazgo transformacional en la salud psicosocial de los empleados. Universitat Jaume I.

Socarrás, Y., Sánchez, J., \& Ucrós, M. (2016). Competencias y Exito Gerencial en Empresas de Servicios Públicos, Municipios de Riohacha Y Maicao, Guajira, Colombia. Sotavento MBA, 27, 132-141.

Solarte, G., \& Martínez, G. (2017). Leadership Styles in men and women in Smes. Ad-Minister, (31), 25-46. https://doi.org/10.17230/ad-minister.31.2

Sperber, S., \& Linder, C. (2018). The impact of top management teams on firm innovativeness: a configurational analysis of demographic characteristics, leadership style and team power distribution. Review of Managerial Science, 12(1), 285-316. https://doi.org/10.1007/s11846-016-0222-z 
Vega, C., \& Zavala, G. (2004). Adaptación del cuestionario multifactorial de liderazgo (MLQ Forma 5 X Corta) de B. Bass y B. Avolio al contexto organizacional chileno. Universidad de Chile.

Vidic, Z., Burton, D., South, G., Pickering, A. M., \& Start, A. (2016). Emotional and Motivational Correlates of Leadership Styles: A Comprehensive Framework for Understanding Effective Leaders. Journal of Leadership Studies, 10(3), 22-40. https://doi.org/10.1002/jls.21485

Villa, A. (2015). Importancia e impacto del liderazgo educativo. Padres Y Maestros, (361). https://doi.org/10.14422/pym.i361.y2015.001

Villacorta, C. (2015). Percepción docente sobre el estilo de liderazgo del subdirector de educación secundaria de la institución educativa particular "Santa Margarita" de Lima. Universidad de Piura.

Villalobos, C., Peña, J., Aguirre, E., \& Guerrero, M. (2017, December). Liderazgo escolar y conflictos socioeducativos. Un estudio exploratorio en liceos públicos chilenos. Revista Calidad En La Educación, 81-111. https://doi.org/https://doi.org/10.31619/caledu.n47.31

Volante, P., Díaz, A., \& Tapia, O. (2003). Proposiciones para la formación en liderazgo educacional. Revista Calidad En La Educación, 239-260. https://doi.org/https://doi.org/10.31619/caledu.n18.401

Wahab, S., Rahmat, A., Yusof, M. S., \& Mohamed, B. (2016). Organization Performance and Leadership Style: Issues in Education Service. Procedia - Social and Behavioral Sciences, 224(August 2015), 593-598. https://doi.org/10.1016/j.sbspro.2016.05.447

Willman, S., \& Velasco, M. (2011). Relación en las percepciones del estilo de liderazgo del jefe inmediato con el desempeño laboral de los estudiantes en práctica de la universidad icesi1. Estudios Gerenciales, 27(118), 67-84. https://doi.org/10.1016/S0123-5923(11)70146-2

Yamak, Ö. U., \& Eyüpoğlu, Ş. Z. (2018). Leadership Styles of Hotel Managers in Northern Cyprus : Which Style is Dominant? AIMI JOURNAL, 7, 1-11. 
PARA CITAR EL ARTÍCULO INDEXADO.

García Puga, S., \& Espinoza Solís, E. (2019). El impacto de los estilos de liderazgo en los centros de educación media del Ecuador, su perspectiva de líderes y docentes en el año 2018. Visionario Digital, 3(3), 134-156. https://doi.org/10.33262/visionariodigital.v3i3.666

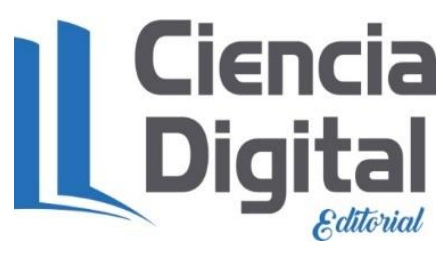

El artículo que se publica es de exclusiva responsabilidad de los autores y no necesariamente reflejan el pensamiento de la Revista Visionario Digital.

El artículo queda en propiedad de la revista y, por tanto, su publicación parcial y/o total en otro medio tiene que ser autorizado por el director de la Revista Visionario Digital.
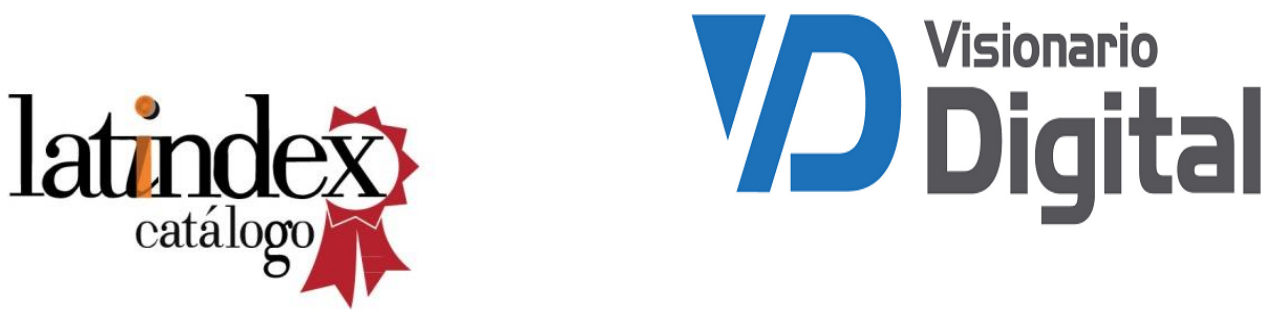